\title{
ORDERABILITY OF TOPOLOGICAL SPACES BY CONTINUOUS PREFERENCES
}

\author{
JOSÉ CARLOS RODRÍGUEZ ALCANTUD
}

(Received 13 March 2001)

\begin{abstract}
We extend van Dalen and Wattel's (1973) characterization of orderable spaces and their subspaces by obtaining analogous results for two larger classes of topological spaces. This type of spaces are defined by considering preferences instead of linear orders in the former definitions, and possess topological properties similar to those of (totally) orderable spaces (cf. Alcantud, 1999). Our study provides particular consequences of relevance in mathematical economics; in particular, a condition equivalent to the existence of a continuous preference on a topological space is obtained.
\end{abstract}

2000 Mathematics Subject Classification. 54F05, 06F30.

1. Introduction. This paper is a natural continuation of a prior contribution in this journal (cf. Alcantud [2]). In that work, we had analyzed a number of significant properties of a class of topologies associated with spaces ordered by preferences (i.e., asymmetric and negatively transitive binary relations). Further issues regarding that framework have been studied in Alcantud [1]. Preferences include the so-called linear or total orders; the analogous study for this particular type of orders had been conducted by different authors before. We now consider a converse problem, which we may situate in the following general framework: which topological spaces can be endowed with an order of a certain type, in such a way that the order and the topology be related in a certain form? Again, we consider the particular case when the order is linear as an initial approach.

To this respect, van Dalen and Wattel [18] solved the problem of characterizing the so-called weakly orderable spaces, orderable spaces, and subspaces of orderable spaces in terms of conditions on a subbase of the space. The technique that was employed in such paper derived from that used by de Groot and Schnare [5]; this later paper was devoted to providing characterizations of compact orderable spaces (which they call totally orderable spaces) and their products. A further improvement of these theorems led to characterizing products of orderable spaces and products of subspaces of orderable spaces (cf. van Dalen [17]). Prior to these achievements, several authors had dealt with these problems (cf. Eilenberg [9], Kok [13, 14], and Herrlich [11] among others). We will extend their study to the case of spaces ordered by preferences. Our purpose is to characterize certain types of topological spaces which generalize weakly orderable and orderable spaces and their subspaces to the preference case.

Apart from its intrinsic interest, this work will enable us to deduce relevant applications to mathematical economics. Indeed, economic agents' (strict) preferences are usually modelled through asymmetric and negatively transitive binary relations. 
The role that preferences play in consumer theory has been exposed, for example, in Debreu [8] and Hildenbrand and Kirman [12]. Some further properties are usually assumed for an agent's preferences, for instance continuity. This requires to work on topological spaces such as Euclidean spaces, a common framework in economic theory. The continuity axiom is not only technical, since it has an intuitive meaning in economic terms. Furthermore, continuous preferences are of particular interest in mathematical utility theory (cf. Bridges and Mehta [4], Debreu [7, Section 4.6], and Rader [16]). The present contribution will enable us to take as a primitive concept the agents' indifference. In particular, we intend to provide necessary and sufficient conditions under which this alternative way to model individual choice behavior leads to that by preferences of certain significant types. Obviously, this will be apparent after studying adequate orderability problems of topological spaces. Some concluding remarks will help to understand these implications better.

2. Some known results. We proceed to fix the notation and list some results that will ease our task.

An asymmetric, negatively transitive binary relation $\prec$ on a set $X$ is a preference. A transitive, asymmetric (or irreflexive) binary relation $\prec$ is called a partial order. With any partial order $\prec$ we may associate a binary relation called indifference relation; it is denoted by $\sim$ and defined by $x \sim y$ if and only if not $x \prec y$, not $y \prec x$. If $\sim$ is associated with a preference, then it is an equivalence relation and conversely. By $x \preceq y$ we mean $x \prec y$ or $x \sim y$. The order topology associated with a partial order $\prec$ (denoted by $\tau_{\text {or }}(\prec)$, or simply $\tau_{\text {or }}$ if no confusion is possible) is the topology which has the family formed by $X$ and all the subsets $\{a \in X: a \prec x\}$ and $\{a \in X: x \prec a\}$ with $x \in X$ as a subbase of open sets. The equivalence classes by an equivalence binary relation $R$ on a set will be denoted by $[x]_{R}$, or simply $[x]$ if no confusion is possible.

The next two definitions were introduced in [2]. Given a collection $\mathscr{S}$ of subsets of a set $X$, a binary relation $R$ on $X$ saturates $\mathscr{Y}$ if $x R y, y \in S$, and $S \in \mathscr{S}$ imply $x \in S$. We say that a quotient map between topological spaces $f:(X, \tau) \rightarrow\left(X^{\prime}, \tau^{\prime}\right)$ is a saturated identification if, for each $A \in \tau, A=f^{-1} f(A)$. We also say that $f: X \rightarrow X^{\prime}$ is a saturated identification with the topologies $\tau, \tau^{\prime}$.

Because an open (closed) continuous onto map is a quotient map, a saturated identification can be redefined as an open (closed) continuous onto map with $A=f^{-1} f(A)$ for all $A \in \tau\left(C=f^{-1} f(C)\right.$ for all $C$ closed). If $f$ is the quotient map associated with an equivalence relation $R$ on $X$, the condition that $A=f^{-1} f(A)$ for all $A \in \tau$ is equivalent to $R$ saturates $\tau$. Thus, for any equivalence binary relation on a topological space that saturates the topology, the projection onto the quotient space is open.

We have proved in [3] the following characterization, which shows that saturation appears naturally in the study of preferences.

THEOREM 2.1. A partial order on a set is a preference if and only if the indifference associated with it saturates its order topology.

In what follows, if a preference on a set $X$ is denoted by $\prec$ then $\sim$ will stand for the indifference that $\prec$ induces. Furthermore, for this preference $\prec$ we denote by $\prec^{\prime}$ the linear order on $X / \sim$ defined in a natural way by $[x] \prec^{\prime}[y]$ if and only if $a \prec b$ 
for all $a \in[x]$ and $b \in[y]$ (cf. [10, Theorem 2.1]). Conversely, if $R$ is an equivalence binary relation on $X$, then any linear order $\prec^{\prime}$ on $X / R$ induces a preference $\prec$ on $X$ in a natural way according to the expression $x \prec y$ if and only if $[x]_{R} \prec^{\prime}[y]_{R}$.

Under these conditions, the following result was proved in Alcantud [2].

Proposition 2.2. If $\prec$ is a preference on $X$ and $\prec^{\prime}$ is the induced linear order on $X / \sim$, then the projection map $p: X \rightarrow X / \sim$ is a saturated identification with the order topologies.

A partial order $\prec$ is compatible with a topology $\tau$ if $\tau_{\text {or }}(\prec) \leqslant \tau$. An alternative term is to say that $\prec$ is continuous with respect to $\tau$.

Weakly orderable spaces are those which can be endowed with a continuous linear order. Orderable spaces are usually called LOTS (for linearly ordered topological space); their subspaces are called GO-spaces (which stands for generalized ordered space). In this paper, we adopt this latter terminology. Thus, a LOTS is a topological space whose topology is induced by a linear order on it. Now, the next definitions are obtained by generalizing the concepts of GO-space and LOTS to the case of spaces ordered by preferences.

DEFINITION 2.3. A GPO-space (for Generalized Preference-Ordered space) is a triple $(X, \prec, \tau)$ where $\prec$ is a preference on $X$ and $\tau$ is a topology on $X$ such that $\tau_{\text {or }} \leqslant \tau$ and $\tau$ has a base formed by convex sets ( $C \subseteq X$ is convex if $p \preceq z \preceq q$ and $p, q \in C$ implies $z \in C)$. A POTS (Preference-Ordered Topological Space) is a triple $\left(X, \prec, \tau_{\text {or }}\right)$ where $\prec$ is a preference on $X$.

These definitions were also introduced in Alcantud [2], which is devoted to the study of those classes of spaces; a study which is complemented in [1]. In particular, we have shown in [2] that GPO-spaces form a class that is contained in the class of monotonically normal spaces (as defined in Kubiak [15]) and that strictly contains GO-spaces. Analogously to the linear case, the class of GPO-spaces coincides with the class of subspaces of POTS. Notice that, in any GPO-space, the underlying indifference saturates the topology.

Following van Dalen and Wattel [18] we say that a nest is a collection $\mathcal{N}$ of sets with the property that for any two members $N_{1}$ and $N_{2}$ of $\mathcal{N}$ it is true that either $N_{1} \subseteq N_{2}$ or $N_{2} \subseteq N_{1}$. It is also common to call this type of collections chains of sets. Besides, a collection $\mathscr{Y}$ of sets is interlocking provided that every set $S_{0}$ of $\mathscr{Y}$ which is an intersection of strictly larger members of $\mathscr{S}$ has a representation as a union of strictly smaller members of $\mathscr{Y}$, that is,

$$
S_{0}=\cap\left\{S: S_{0} \subseteq S, S \in \mathscr{S} \backslash\left\{S_{0}\right\}\right\} \Longrightarrow S_{0}=\cup\left\{S: S \subseteq S_{0}, S \in \mathscr{S} \backslash\left\{S_{0}\right\}\right\} .
$$

Then, the following theorem was proved in [18].

TheOREM 2.4 (van Dalen and Wattel [18]). A $T_{1}$ topological space is a GO-space (resp., a LOTS) if and only if it has an open subbase consisting of two nests (resp., of two interlocking nests). A topological space admits a continuous linear order on it if and only if there exist two nests of open sets that together generate a $T_{1}$ topology. 
We point out that interlocking nests appear naturally in the study of the spaces ordered by continuous preferences. A straightforward argument shows that, for any $\prec$ continuous preference on $X$, the collection of all the subsets $x^{u}:=\{y \in X: x \prec y\}$ (resp., $x^{l}:=\{y \in X: y \prec x\}$ ) with $x$ ranging over $X$ is an interlocking nest. These sets are called upper (resp., lower) contour sets associated with $x$ in the economic literature.

A different solution to the problem of characterizing LOTS and GO-spaces was stated by Deák in [6], by using the concept of compatible directions.

3. Orderability of topological spaces by continuous preferences. We begin by obtaining a basically technical result, for the sake of the exposition.

THEOREM 3.1. Let $(X, \tau)$ be a topological space and let $R$ be an equivalence binary relation on $X$. The following statements are equivalent:

(a) There exists a preference $\prec$ on $X$ whose indifference coincides with $R$ and such that $(X, \prec, \tau)$ is a GPO-space.

(b) $R$ saturates $\tau$, the equivalence classes by $R$ are closed and $\tau$ has a subbase constituted by the union of two nests.

Proof. (a) $\Rightarrow(b)$. It is well known that, in any space ordered by a preference, the equivalence classes induced by its indifference are closed in its order topology. Besides, the indifference $R$ saturates $\tau$ by the convexity of the open sets, and the nests of open subsets $\left\{x^{u}: x \in X\right\}$ and $\left\{x^{l}: x \in X\right\}$ fulfill the remaining requirement.

(b) $\Rightarrow$ (a). Suppose that $\tau$ has a subbase which is constituted by the union of two nests $U$ and $\mathscr{L}$. The projection $p$ onto the quotient space by $R$ is open because $R$ saturates $\tau$. We show that $p(U)$ and $p(\mathscr{L})$ are two nests of open sets whose union generates $\tau_{\text {quot }}$, which is $T_{1}$.

It is clear that $p(U)$ and $p(\mathscr{L})$ are nests of open sets. Now, let $A \in \tau_{\text {quot }}$ and $x^{\prime} \in A$. Take an element $x \in p^{-1}\left(x^{\prime}\right)$; there must exist $\left\{A_{i}: i=1, \ldots, n\right\} \subseteq \mathcal{U}$ and $\left\{B_{j}: j=\right.$ $1, \ldots, m\} \subseteq \mathscr{L}$ such that

$$
x \in \cap\left\{A_{i}: i=1, \ldots, n\right\} \cap\left\{B_{j}: j=1, \ldots, m\right\} \subseteq p^{-1}(A) .
$$

Since $R$ saturates $\tau$, it follows that $p\left(\cap\left\{A_{i}: i=1, \ldots, n\right\} \cap\left\{B_{j}: j=1, \ldots, m\right\}\right)=$ $\cap\left\{p\left(A_{i}\right): i=1, \ldots, n\right\} \cap\left\{p\left(B_{j}\right): j=1, \ldots, m\right\}$. Thus

$$
\begin{aligned}
x^{\prime} \in & \cap\left\{p\left(A_{i}\right): i=1, \ldots, n\right\} \cap\left\{p\left(B_{j}\right): j=1, \ldots, m\right\} \\
& =p\left(\cap\left\{A_{i}: i=1, \ldots, n\right\} \cap\left\{B_{j}: j=1, \ldots, m\right\}\right) \subseteq p p^{-1}(A)=A .
\end{aligned}
$$

This shows that the union of $p(U)$ and $p(\mathscr{L})$ is a subbase for $\tau_{\text {quot }}$.

From Theorem 2.4, there exists a linear order $\prec^{\prime}$ on $X / R$ verifying that $\tau_{\text {or }}\left(\prec^{\prime}\right) \leqslant$ $\tau_{\text {quot }}$ and such that the open sets of a base $\mathscr{B}^{\prime}$ of $\tau_{\text {quot }}$ are convex respect to $\prec^{\prime}$. Let $\prec$ be the preference on $X$ that induces $\prec^{\prime}$ on $X / R$.

By Proposition 2.2, the projection $p$ is a saturated identification with $\tau_{\text {or }}(\prec)$ and $\tau_{\text {or }}\left(\prec^{\prime}\right)$, and thus $A \in \tau_{\text {or }}(\prec)$ implies $p(A) \in \tau_{\text {or }}\left(\prec^{\prime}\right)$, therefore $p(A) \in \tau_{\text {quot }}$. Since $R$ saturates $\tau_{\text {or }}(\prec)$ we deduce from $A \in \tau_{\text {or }}(\prec)$ that $A=p^{-1} p(A) \in \tau$. Therefore $\tau_{\text {or }}(\prec) \leqslant \tau$. 
On the other hand, the collection $\mathscr{B}=\left\{p^{-1}\left(B^{\prime}\right): B^{\prime} \in \mathscr{B}^{\prime}\right\}$ is a base of $\tau$ because $p$ is a saturated identification with $\tau$ and $\tau_{\text {quot }}$. This collection is formed by convex sets with respect to the preference $\prec$, which permits to conclude that $(X, \prec, \tau)$ is a GPO-space.

We now proceed to characterize POTS with regards to stating necessary and sufficient conditions for a given indifference to derive from a model by continuous preferences of a particular form.

THEOREM 3.2. Let $(X, \tau)$ be a topological space and let $R$ be an equivalence binary relation on $X$. The following statements are equivalent:

(a) There exists a preference $\prec$ on $X$ whose indifference coincides with $R$ and such that $(X, \prec, \tau)$ is a POTS.

(b) $R$ saturates $\tau$, the equivalence classes by $R$ are closed and $\tau$ has a subbase constituted by the union of two interlocking nests.

REMARK 3.3. Notice that a particular interpretation may be derived if $R$ is thought of as an agent's indifference. In this case, condition (b) of Theorem 3.2 is equivalent to the existence of a preference on $X$ which induces the indifference $R$ and whose order topology coincides with the original topology.

Proof OF Theorem 3.2. Again, the implication $(\mathrm{a}) \Rightarrow(\mathrm{b})$ is immediate.

(b) $\Rightarrow$ (a). Suppose that $\tau$ has a subbase that is constituted by the union of two interlocking nests, namely $U$ and $\mathscr{L}$. The projection $p$ onto the quotient space by $R$ is open because $R$ saturates $\tau$. As in Theorem 3.1, $p(U)$ and $p(\mathscr{L})$ are two nests of open subsets that generate $\tau_{\text {quot }}$, which is $T_{1}$. We show that $p(\mathscr{Y})$ is interlocking for each $\mathscr{Y}$ interlocking collection of subsets of $X$ such that $R$ saturates $\mathscr{Y}$, which implies that $p(U)$ and $p(\mathscr{L})$ are interlocking.

Assume that $S_{0}$ is a set of $\mathscr{S}$ such that

$$
p\left(S_{0}\right)=\cap\left\{p(S): p\left(S_{0}\right) \subseteq p(S), p(S) \in p(\mathscr{S}) \backslash\left\{p\left(S_{0}\right)\right\}\right\} .
$$

Since $R$ saturates $\mathscr{Y}$,

$$
p^{-1} p\left(S_{0}\right)=\cap\left\{S: p\left(S_{0}\right) \subseteq p(S), p(S) \in p(\mathscr{S}) \backslash\left\{p\left(S_{0}\right)\right\}\right\} .
$$

Now $\left\{S: p\left(S_{0}\right) \subseteq p(S), p(S) \in p(\mathscr{S}) \backslash\left\{p\left(S_{0}\right)\right\}\right\}=\left\{S: S_{0} \subseteq S, S \in \mathscr{S} \backslash\left\{S_{0}\right\}\right\}$, which will permit to express $S_{0}=p^{-1} p\left(S_{0}\right)=\cap\left\{S: S_{0} \subseteq S, S \in \mathscr{S} \backslash\left\{S_{0}\right\}\right\}$. Because $\mathscr{Y}$ is interlocking we can express $S_{0}=\cup\left\{S: S \subseteq S_{0}, S \in \mathscr{S} \backslash\left\{S_{0}\right\}\right\}$. From this it follows that $p\left(S_{0}\right)=$ $\cup\left\{p(S): S \subseteq S_{0}, S \in \mathscr{S} \backslash\left\{S_{0}\right\}\right\}$. We may now conclude the argument because

$$
\left\{p(S): p(S) \subseteq p\left(S_{0}\right), p(S) \in p(\mathscr{Y}) \backslash\left\{p\left(S_{0}\right)\right\}\right\}=\left\{p(S): S \subseteq S_{0}, S \in \mathscr{Y} \backslash\left\{S_{0}\right\}\right\},
$$

and therefore $p\left(S_{0}\right)=\cup\left\{p(S): p(S) \subseteq p\left(S_{0}\right), p(S) \in p(\mathscr{Y}) \backslash\left\{p\left(S_{0}\right)\right\}\right\}$.

From Theorem 2.4, there exists a linear order $\prec^{\prime}$ on $X / R$ such that $\tau_{\text {quot }}=\tau_{\text {or }}\left(\prec^{\prime}\right)$. Let $\prec$ be the preference on $X$ that induces $\prec^{\prime}$ on $X / R$; we show that $\tau=\tau_{\text {or }}(\prec)$.

Indeed, from Proposition 2.2 the projection $p$ is a saturated identification with $\tau_{\text {or }}(\prec)$ and $\tau_{\text {or }}\left(\prec^{\prime}\right)$; therefore, from $A \in \tau_{\text {or }}(\prec)$ we deduce $p(A) \in \tau_{\text {or }}\left(\prec^{\prime}\right)$, or, equivalently, $p(A) \in \tau_{\text {quot }}$. Besides, $R$ saturates $\tau_{\text {or }}(\prec)$ because it is the indifference associated with $\prec$, thus $A \in \tau_{\text {or }}(\prec) \Rightarrow p^{-1} p(A)=A$. 
It follows that $\tau_{\text {or }}(\prec) \leqslant \tau$ because

$$
A \in \tau_{\text {or }}(\prec) \Longrightarrow p(A) \in \tau_{\text {quot }} \Longrightarrow p^{-1} p(A) \in \tau \Longrightarrow A \in \tau .
$$

On the other hand, if $A \in \tau$ then $p(A) \in \tau_{\text {quot }}$, and now $\tau_{\text {or }}\left(\prec^{\prime}\right)=\tau_{\text {quot }}$ yields $p^{-1} p(A) \in \tau_{\text {or }}(\prec)$ because $p:\left(X, \tau_{\text {or }}(\prec)\right) \rightarrow\left(X / R, \tau_{\text {or }}\left(\prec^{\prime}\right)\right)$ is continuous (Proposition 2.2). From $A \in \tau$ we obtain that $A=p^{-1} p(A)$ because $R$ saturates $\tau$, and thus $A \in \tau$ yields $p^{-1} p(A) \in \tau_{\text {or }}(\prec)$ and so $A \in \tau_{\text {or }}(\prec)$. Therefore $\tau \leqslant \tau_{\text {or }}(\prec)$. This ends the proof.

Finally, we prove the following characterization of the topological spaces that may be ordered by a continuous preference. This will yield the main application to individual choice behavior, and particularly to mathematical economics.

THEOREM 3.4. Let $(X, \tau)$ be a topological space and let $R$ be an equivalence binary relation on $X$. The following statements are equivalent:

(a) There exists a preference on $X$ which is continuous with respect to $\tau$ and whose indifference coincides with $R$.

(b) There exist two nests of open sets of $\tau$ whose union generates a topology $\tau^{\prime}$ such that $R$ saturates $\tau^{\prime}$ and the equivalence classes by $R$ are closed in $\tau^{\prime}$.

REMARK 3.5. Again, we may reinterpret this result by taking $R$ as an agent's indifference. We thus obtain a condition which is equivalent to the existence of a continuous preference on $X$ inducing $R$ as its indifference. Therefore, if we depart from a model of individual choice behavior in which indifference is a primitive concept, then we know under which conditions this is compatible with assuming that there are underlying continuous preferences explaining such behavior.

Proof of Theorem 3.4. The implication $(\mathrm{a}) \Rightarrow(\mathrm{b})$ is immediate.

(b) $\Rightarrow$ (a). As $p:\left(X, \tau^{\prime}\right) \rightarrow\left(X / R,\left(\tau^{\prime}\right)_{\text {quot }}\right)$ is open, then the elements of $p(\mathcal{U})$ and $p(\mathscr{L})$ are open in $\tau_{\text {quot }}$. As in Theorem 3.1, $p(U)$ and $p(\mathscr{L})$ are two nests whose union generates $\left(\tau^{\prime}\right)_{\text {quot }}$, which is $\mathrm{T}_{1}$. From Theorem 2.4 there exists a linear order $\prec^{\prime}$ on $X / R$ that is compatible with $\tau_{\text {quot }}$.

Let $\prec$ be the preference on $X$ whose indifference coincides with $R$ and naturally induces $\prec^{\prime}$ on $X / R$. Then, the fact that the indifference $R$ associated with $\prec$ saturates the order topology $\tau_{\text {or }}(\prec)$ and Proposition 2.2 yield that $\prec$ is the desired preference.

4. Concluding remarks. We have provided new orderability results that permit immediate applications to fields like consumer theory. A closer look at the implications in this field may be of interest for non-specialists.

The primitive concept in consumer theory is typically the binary relation that represents the agent's tastes. The classical assumptions include asymmetry and negative transitivity, which have been long criticized since they impose a "too rational" behavior. It is well known that the indifference associated with an asymmetric and negatively transitive binary relation (i.e., a preference) is an equivalence relation. Therefore, one might be interested in taking indifference as the primitive concept in the model instead. That would permit more general approaches, since it does not presuppose as much rationality on the agent as requesting that he/she has expressed 
tastes by preferences. One may pose the following natural, related problems. Firstly, when is the knowledge of indifference equivalent to the knowledge of the preference? Secondly, a crucial question regarding the relationship between the two approaches in both directions. On the one hand, it is obvious that modeling through indifference is more general than proceeding by continuous preferences, since it allows for many more possibilities. On the other hand, and concerning the converse problem, assume now that an agent has some indifference defined on a commodity space on which a topology has been fixed; when his/her tastes can be modeled by a continuous preference (possibly satisfying further requirements) yielding that indifference and therefore explaining the original choice behavior? Needless to say, indifference must be an equivalence relation because of the observation above, and thus we may speak of proper indifference classes.

With regards to the first question, we have proved the following result. If the commodity space is connected and the consumer's preferences are assumed to be continuous, then the indifference determines the preference up to inversion (cf. Alcantud and Gutiérrez [3]). In the usual classroom situation where the commodity space is a connected subset of $\mathbb{R}^{n}$, monotonicity would determine the preference univocally in a natural way.

The second question has been answered as a natural application of the results herein contained. This construction paves the way towards developing a consumer theory on the basis of the concept of indifference. An adequate model would permit us to proceed, under the conditions required by the corresponding orderability results, as if the agent's behavior was determined by continuous preferences, which allows the researcher to apply the extensive theoretical results for that model. The predictive capabilities under the knowledge of the weaker concept of indifference only can be, therefore, significantly enhanced.

AcKNOwLEDGEMENT. This research was supported by DGICYT Project PB98-551.

\section{REFERENCES}

[1] J. C. R. Alcantud, Topological separability and axioms of countability in GPO-spaces, Bull. Austral. Math. Soc. 55 (1997), no. 1, 131-142. MR 98b:54043. Zbl 903.54013.

[2] _ , Topological properties of spaces ordered by preferences, Int. J. Math. Math. Sci. 22 (1999), no. 1, 17-27. MR 2000f:06016. Zbl 924.06016.

[3] J. C. R. Alcantud and J. M. Gutiérrez, Preference through indifference: a topological approach, J. Math. Econom. 31 (1999), no. 4, 543-551. MR 99m:90012.

[4] D. S. Bridges and G. B. Mehta, Representations of Preferences Orderings, Lecture Notes in Economics and Mathematical Systems, vol. 422, Springer-Verlag, Berlin, 1995. MR 96c:90006. Zbl 836.90017.

[5] J. de Groot and P. S. Schnare, A topological characterization of products of compact totally ordered spaces, General Topology and Appl. 2 (1972), 67-73. MR 45\#9290. Zbl 254.54006.

[6] E. Deák, Theory and applications of directional structures, Topics in Topology (Proc. Colloq., Keszthely, 1972), Colloq. Math. Soc. Janos Bolyai, vol. 8, North-Holland, Amsterdam, 1974, pp. 187-211. MR 52\#11860. Zbl 355.54007.

[7] G. Debreu, Representation of a preference ordering by a numerical function, Decision Processes (R. M. Thrall, C. H. Coombs, and R. L. Davis, eds.), John Wiley, New York, 1954, pp. 159-165. Zbl 0058.13803. 
[8] _ Theory of Value: an Axiomatic Analysis of Economic Equilibrium, Cowles Foundation for Research in Economics at Yale University, Monograph, vol. 17, John Wiley \& Sons, New York, 1959. MR 22\#1447. Zbl 193.20205.

[9] S. Eilenberg, Ordered topological spaces, Amer. J. Math. 63 (1941), 39-45. MR 2,179e. Zbl 024.19203.

[10] P. C. Fishburn, Interval Orders and Interval Graphs. A Study of Partially Ordered Sets, Wiley-Interscience Series in Discrete Mathematics, John Wiley \& Sons, Chichester, 1985. MR 86m:06001. Zbl 551.06001.

[11] H. Herrlich, Ordnungsfähigkeit Topologischer Räume, Inaugural-Dissertation zur Erlangung der Doktorwürde der Mathematisch-Naturwissenschaftlichen Fakultät der Freien Universität Berlin, Berlin, 1962 (German). MR 27\#4196.

[12] W. Hildenbrand and A. P. Kirman, Equilibrium Analysis. Variations on Themes by Edgeworth and Walras, Advanced Textbooks in Economics, vol. 28, North-Holland Publishing, Amsterdam, 1988. MR 90m:90064. Zbl 666.90001.

[13] H. Kok, On conditions equivalent to the orderability of a connected space, Nieuw Arch. Wisk. (3) 18 (1970), 250-270. MR 45\#9295. Zbl 202.21803.

[14]_ Connected Orderable Spaces, Mathematical Centre Tracts, no. 49, Mathematisch Centrum, Amsterdam, 1973. MR 49\#3862. Zbl 276.54031.

[15] T. Kubiak, Monotone insertion of continuous functions, Questions Answers Gen. Topology 11 (1993), no. 1, 51-59. MR 94a:54048. Zbl 826.54023.

[16] T. Rader, The existence of a utility function to represent preferences, Rev. Econom. Stud. 30 (1963), 229-232.

[17] J. van Dalen, A characterization of products of totally ordered spaces, General Topology and Appl. 4 (1974), 95-101. MR 49\#6181. Zbl 283.54016.

[18] J. van Dalen and E. Wattel, A topological characterization of ordered spaces, General Topology and Appl. 3 (1973), 347-354. MR 49\#6182. Zbl 272.54026.

JosÉ CARLOS RODRÍGUEZ ALCANTUD: FACULTAD DE ECONOMIA Y EMPRESA, UNIVERSIDAD DE SALAMANCA, 37008 SALAMANCA, SPAIN

E-mail address: jcr@usal.es 


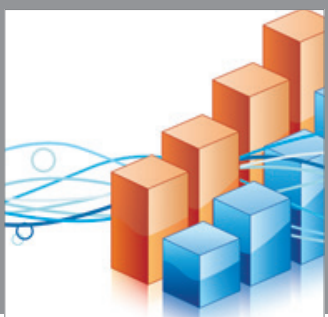

Advances in

Operations Research

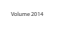

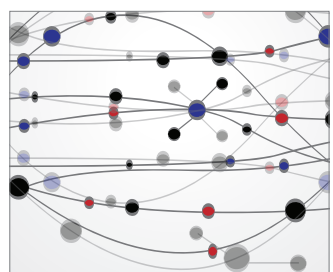

\section{The Scientific} World Journal
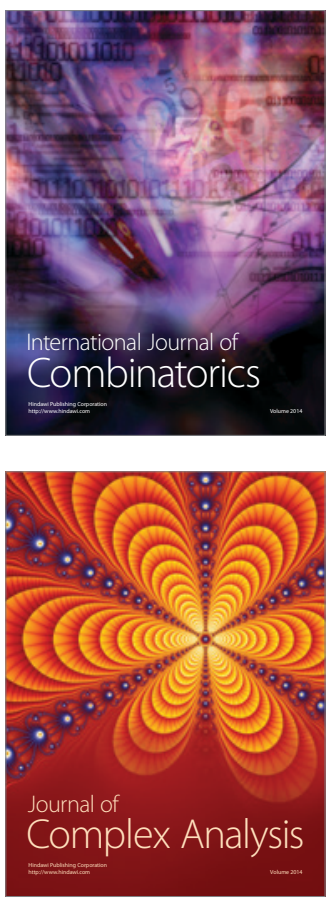

International Journal of

Mathematics and

Mathematical

Sciences
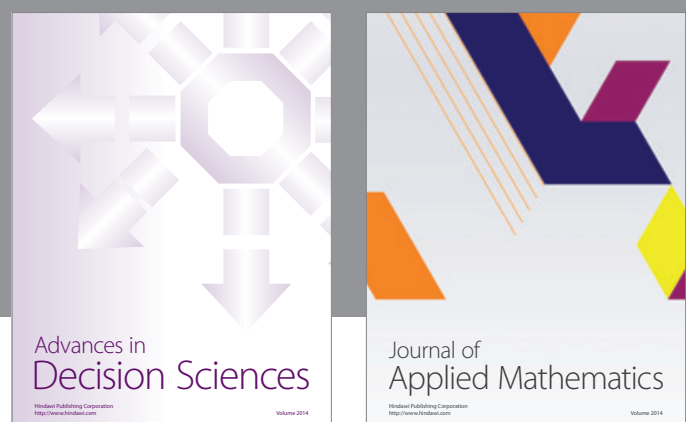

Journal of

Applied Mathematics
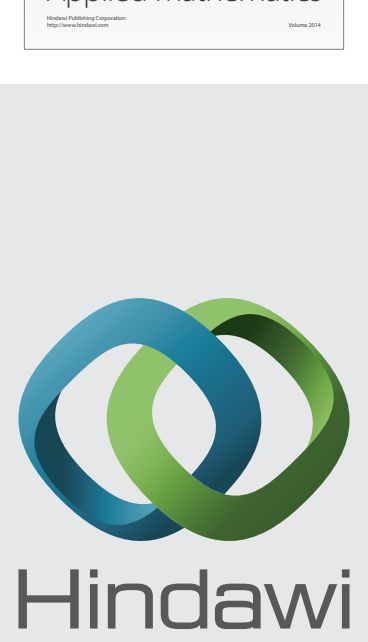

Submit your manuscripts at http://www.hindawi.com
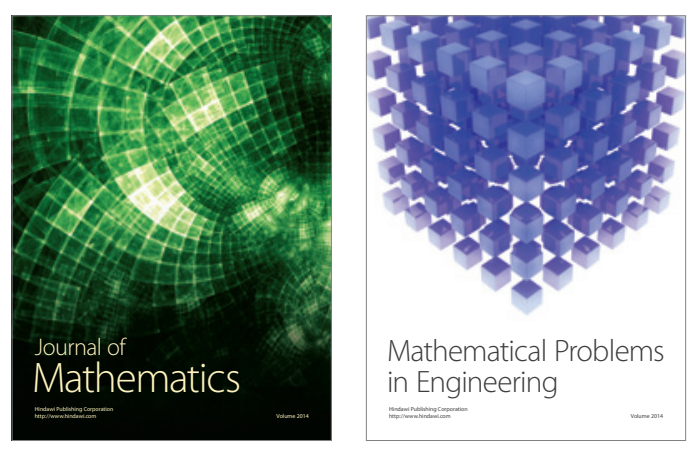

Mathematical Problems in Engineering
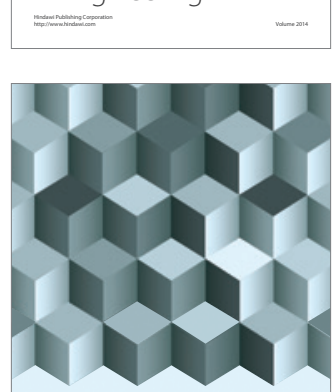

Journal of

Function Spaces
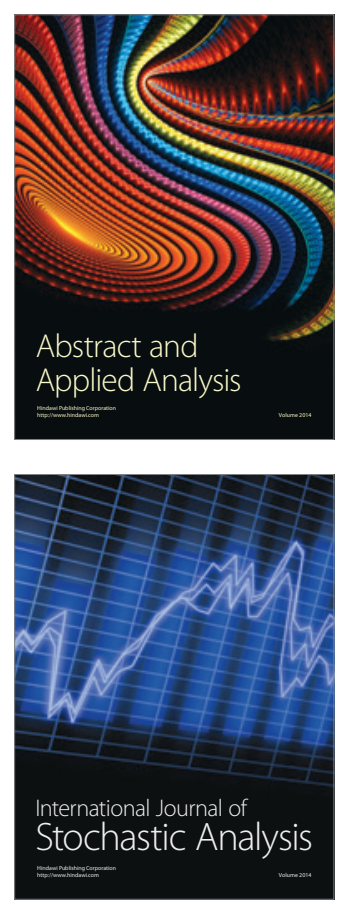

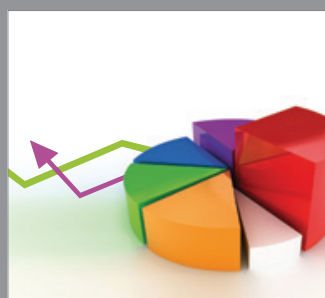

ournal of

Probability and Statistics

Promensencen
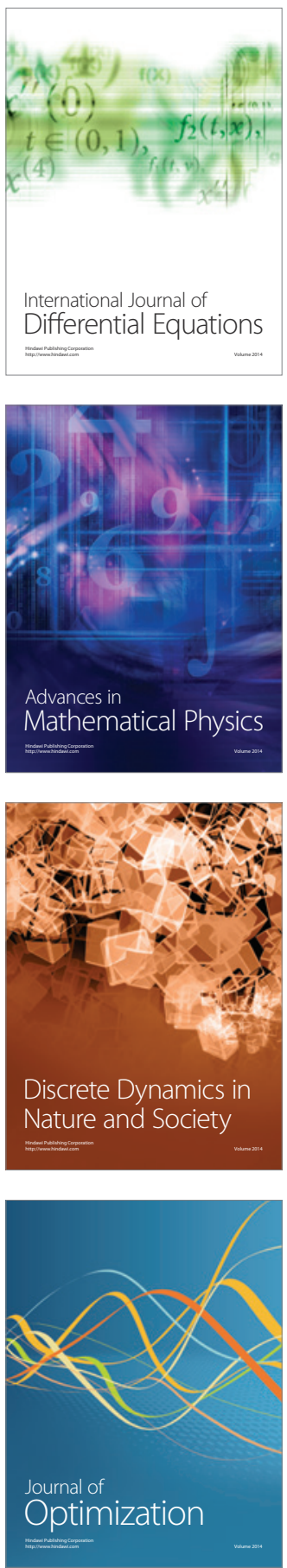